\title{
Design of an Obstruction-Free Phase-Shifter Yielding Maximum Contrast Over a Large Range of Spatial Frequencies
}

\section{H.H. Rose}

\section{Institute of Applied Physics, TU Darmstadt, Hochschulstrasse 6, 64289 Darmstadt, Germany}

The incorporation of an obstruction-free phase plate into the transmission electron microscope and the use of low voltages increase significantly the contrast of low- $Z$ material. We propose a novel phase plate which shifts the phase over a large range of spatial frequencies. Two classes of materials will benefit from using low voltages for image formation. The first class concerns solid materials, such as nanotubes. These objects will not be damaged at voltages below the threshold for surface etching. Moreover, the objects will be imaged with very high contrast at these voltages. The second class of materials comprises insulators and organic materials. For these objects two properties are in concurrence when going to lower voltages: (a) the increase in radiation damage for fixed dose and (b) the increase in contrast. It is experimentally shown that with decreasing voltage, contrast is increasing faster than damage because we need an appreciably lower dose for obtaining the same $\mathrm{S} / \mathrm{N}$ [1]. Therefore, the attainable specimen resolution of radiation-sensitive objects will improve when going to low voltages provided that the object thickness remains smaller than the mean free path length of the electrons.

The incorporation of an adjustable electrostatic potential is a very promising method for realizing Zernike-type phase contrast. Scherzer proposed an obstruction-free phase shift of the scattered electron wave by utilizing the spherical aberration of the objective lens together with a properly chosen defocus $\Delta f=\Delta f_{S}=\sqrt{\lambda} C_{3}$ known as Scherzer focus [2]. The Scherzer phase shift $\chi_{s}$ represents roughly an annular Zernike phase plate, which shifts the phase of the scattered wave by about $-\pi / 2$ for spatial frequencies $q$ within the range $q_{\min } \leq q \leq q_{\max }$. Unfortunately, the useful relative width $q_{\max } / q_{\min }$ of the phase plate is only about 4 . In order to increase this domain significantly and to achieve negative phase contrast, we need a phase shift $\pi / 2$ and a ratio $q_{\max } / q_{\min } \geq 100$. We can realize these requirements in an aberration-corrected TEM by placing a proper phase shifter in the region behind the first intermediate image of the object whose magnification $M$ must be chosen appropriately.

In order to avoid obstruction by the field-forming electrodes, it is advantageous to perform the phase shift at anamorphotic images of the diffraction plane [3]. Such strongly first-order distorted stigmatic images can only be obtained by an appropriate quadrupole system, which enlarges the diffraction pattern in one direction and compresses it in the orthogonal direction. The line-shaped images enable us to shift the phase of the scattered wave without any obstruction by means of micro phase plates depicted in Fig. 1. The adjustable electric potential of the phase plate can be considered as the superposition of the electric potential of a quadrupole with that of a cylinder lens, such that the resulting potential has a saddle point at the centre of the phase plate. Our investigations have shown that ratios $q_{\max } / q_{\min } \geq 100$ are achievable by means of a quadrupole system. It represents in combination with the phase plate an obstruction-free phase-shifter. The suitable quadrupole system shown in Fig. 2 consists of eight quadrupoles which form two orthogonal anamorphotic images. A phase plate is placed at each of the two images. Each of the two phase plates introduces a phase shift of $\pi / 4$ between the scattered wave and the non-scattered wave except for scattering directions which 
are roughly perpendicular to the long side of the anamorphotic image. However, we can tolerate this shortcoming because it reduces the phase contrast by less than $30 \%$ only in very narrow bands along the $x$ - and $y$-axis, as illustrated in Fig. 3.

References

[1] M.T. Hayashida et al., Nuclear Inst. and Meth. in Phys. Res. B 248 (2006) 273.

[2] O. Scherzer, J. Appl. Phys. 20 (1949) 20.

[3] R. Schroeder, B. Barton, H. Rose, G. Benner, Microscc. Microanal. 13 (Suppl. 3) (2007) 008.

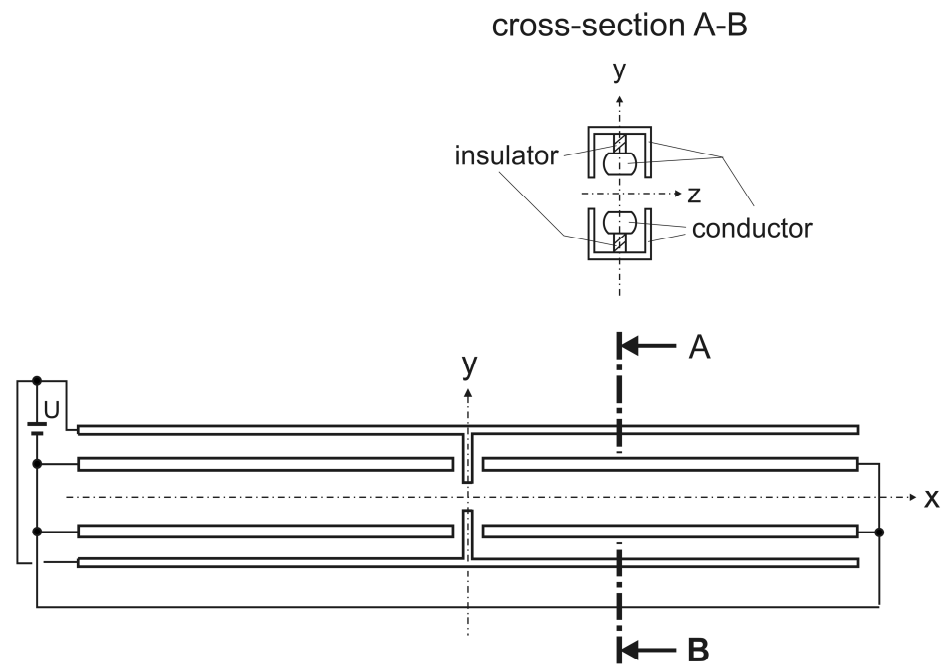

FIG. 1 Scheme of the obstruction-free phase plate, $U$ is the voltage between the outer and inner conductors.

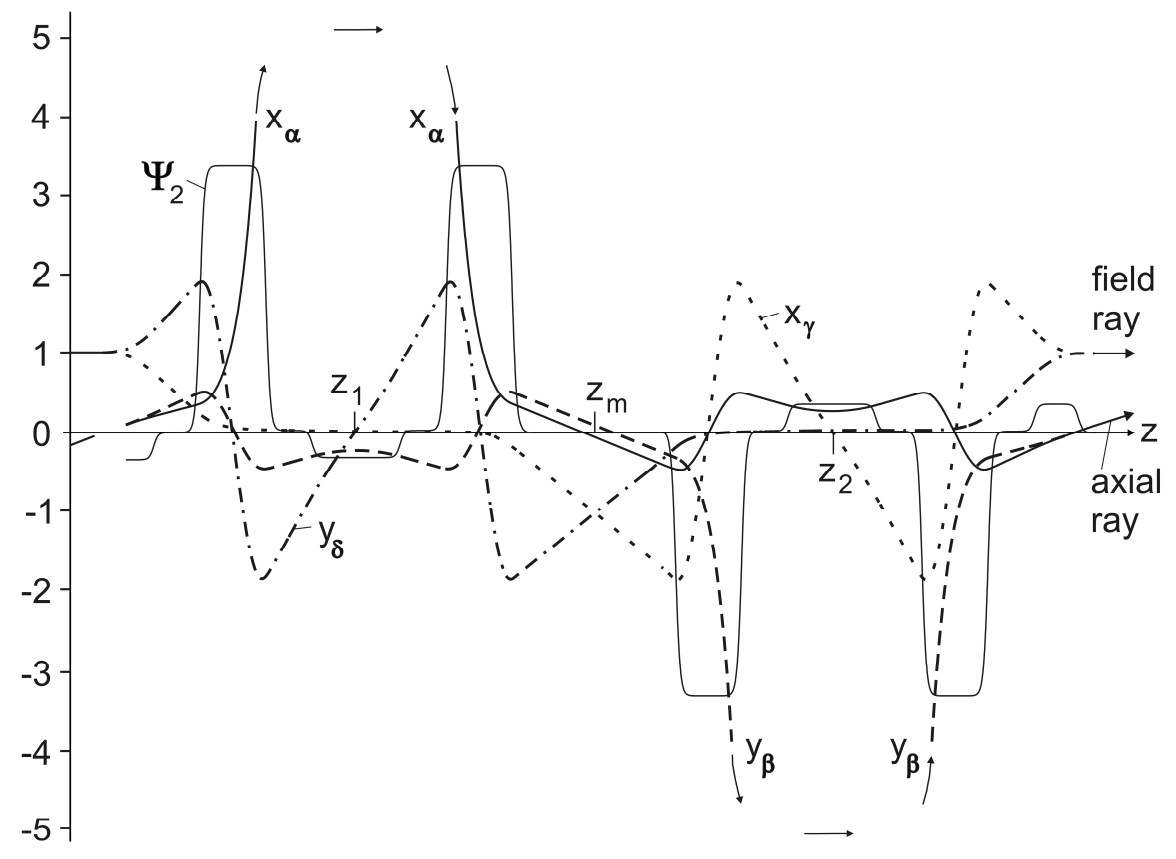

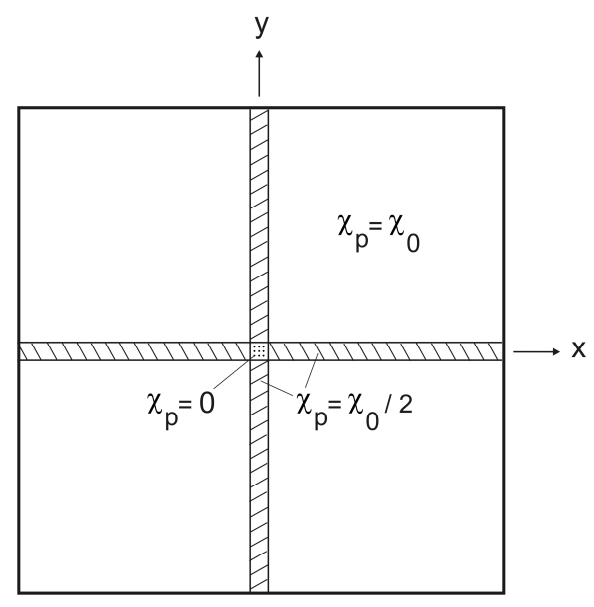

FIG. 3 Total phase shift introduced by the phase shifter.
FIG. 2

Course $\Psi_{2}=\Psi_{2}(z)$ of the axial magnetic quadrupole strength and course of the field rays $x_{\gamma}, y_{\delta}$ and the axial rays $x_{\alpha}, y_{\beta}$, respectively, along the optic axis within the phase shifter. Anamorphotic images of the diffraction plane are located at the planes $z_{1}$ and $z_{2}$ where the phase plates are placed. 